\title{
Urological Evaluation of Tethered Cord Syndrome
}

\author{
Kwanjin Park \\ Department of Urology, Seoul National University Children's Hospital, Seoul National University College of Medicine, Seoul, Korea
}

To describe how to perform urological evaluation in children with tethered cord syndrome (TCS). Although a common manifestation of TCS is the development of neurogenic bladder in developing children, neurosurgeons often face difficulty in detecting urological problems in patients with TCS. From a urological perspective, diagnosis of TCS in developing children is further complicated due to the differentiation between neurogenic bladder dysfunctions and transient bladder dysfunctions owing to developmental problems. Due to the paucity of evidence regarding evaluation prior to and after untethering, I have shown the purpose and tools for evaluation in my own practice. This may be tailored to the types of neurogenic bladder, developmental status, and risks for deterioration. While the urodynamic study (UDS) is the gold standard test for understanding bladder function, it is not a panacea in revealing the nature of bladder dysfunction. In addition, clinicians should consider the influence of developmental processes on bladder function. Before untethering, UDS should reveal synergic urethral movement, which indicates an intact sacral reflex and lack of TCS. Postoperatively, the measurement of post-void residual urine volume is a key factor for the evaluation of spontaneous voiders. In case of elevation, fecal impaction, which is common in spinal dysraphism, should be addressed. In patients with clean intermittent catheterization, the frequency-volume chart should be monitored to assess the storage function of the bladder. Toilet training is an important sign of maturation, and its achievement should be monitored. Signs of bladder deterioration should be acknowledged, and follow-up schedule should be tailored to prevent upper urinary tract damage and also to determine an adequate timing for intervention. Neurosurgeons should be aware of urological problems related to TCS as well as urologists. Cooperation and regular discussion between the two disciplines could enhance the quality of patient care. Accumulation of experience will improve follow-up strategies.

Key Words : Neural tube defects · Urodynamics · Spinal dysraphism · Urinary bladder, Neurogenic.

\section{INTRODUCTION}

Spinal dysraphism (SD; often referred to as spina bifida) includes myriads of abnormal conditions of the spinal cord. It can be seen from the overt protrusion of the spinal cord to normal looking back in the case of occult spina bifida. If left untreated, some of the affected patients may suffer progressive deterioration in the lower extremity and bladder.

This constellation of clinical symptoms or signs, whether neurological, orthopedic, or urological, which may result from SD is referred to as tethered cord syndrome (TCS) ${ }^{6}$. Untethering procedures of the spinal cord performed by the neurosurgeons are expected to halt progression of TCS and further neurological deficits; however it does not always cause the

- Received : March 10, 2020 •Revised : April 22, 2020 •Accepted : April 23, 2020

- Address for reprints : Kwanjin Park

Department of Urology, Seoul National University Children's Hospital, 101 Daehak-ro, Jongno-gu, Seoul 03080, Korea

Tel : +82-2-2072-0695, Fax : +82-2-742-4665, E-mail : urodori@naver.com, urodori9@snu.ac.kr, ORCID : https://orcid.org/0000-0002-8926-3047

This is an Open Access article distributed under the terms of the Creative Commons Attribution Non-Commercial License (http://creativecommons.org/licenses/by-nc/4.0) which permits unrestricted non-commercial use, distribution, and reproduction in any medium, provided the original work is properly cited. 
reversal of an already occurring neurological deficit. Since there is no specific study in diagnosing TCS, proper diagnosis is important for timely surgery at the early stage of TCS and for avoiding unnecessary surgery for those who are not at risk of TCS.

Urologists attending to these patients should have sufficient knowledge of TCS and have adequate level of communication with neurosurgeons. Since neurosurgeons often have limited knowledge about urological ailments that occur during the release of TCS, urologists should counsel the patients and parents about these problems on their behalf. Unless urologists and neurosurgeons communicate well, detection of several important urological manifestations that neurosurgeons must be concerned about might be missed.

Since SD reflects neurological malformation where urological function is controlled, it might be associated with several urological issues. Certain symptoms might also occur due to the neural damage during the untethering procedure. Finally, progressive urological problems occur due to retethering of the spinal cord (also known as secondary TCS). Urologists can play a crucial role in detecting, differentiating, and addressing these problems.

Therefore, the purpose of urological investigation in these patients is to evaluate whether the bladder would deteriorate and to determine an adequate strategy to address this condition.

In this paper, urological investigation of patients with TCS is described. This investigation might vary according to the developmental and functional status of the patients and their risk of deterioration. Since such individual risk stratification has not been well defined, some follow-up schedules ${ }^{6,8}$ that have been recommended might not be applicable for others. In addition, this is not the main concern for neurosurgeons who would be the main readers of the paper. In this paper, I introduced the concept of urological follow-up. For better understanding, the tools employed for urological evaluation have been explained, followed by a description of key points that need to be checked during preoperative, immediate postoperative, and prolonged postoperative follow-up periods, including toilet training.

\section{UROLOGICAL TOOLS FOR EXAMINATION OF PATIENTS WITH SD}

\section{History}

History of the patient is an important element in such evaluation. This includes urological history, such as storage and emptying function, toilet training, urinary tract infection, and urinary tract anomalies. General developmental history and severity of SD (level of conus, presence of syrinx, amount, and location of lipoma) must be taken into account. Since the bladder and bowel, at least partially, share the neurological circuit and are located in close proximity, the history related to the bowel also provides crucial information regarding the neurodevelopment of patients.

\section{Frequency volume chart}

In the patients who have completed the toilet training, the frequency volume chart reveals the time of voiding and the voided volume. In addition, it is useful for evaluation of storage function in patients undergoing clean intermittent catheterization (CIC). It could indicate the overall storage function of the bladder and provide insight regarding the sensory function of the bladder. Usually, the patients are asked to fill up for at least 2 days to obtain meaningful data. It would be better to include the amount of patient's intake (referred to as voiding diary) and 7 days of defecation history as a reference when considering bladder drilling in the context of behavioral treatment ${ }^{1)}$.

\section{Postvoid residual urine volume measurement}

In the case of neurologically intact spontaneous voiders, the amount of postvoid residual urine (PVR urine) could be an adequate marker of the efficiency of voiding ${ }^{2)}$. Adequate sphincteric function is crucial for the initiation and completion of voiding. However, in the presence of sphincteric dysfunction, failure of sphincteric relaxation or even active sphincteric contraction during detrusor contraction (detrusor sphincter dyssynergia, DSD) leads to incomplete emptying of the bladder. This reflects the situation that is observed in cases of TCS, where DSD can occur as a manifestation of upper motor neuron disease. For children 4 to 6 years of age, a single PVR greater than $30 \mathrm{~mL}$, or greater than $21 \%$ of bladder capacity, or repetitive PVR $>20 \mathrm{~mL}$, or greater than $10 \%$ of bladder capacity, can be regarded as elevated PVR. For children 7 
to 12 years, a single PVR greater than $20 \mathrm{~mL}$, or $15 \%$ of bladder capacity, or repetitive PVR greater than $10 \mathrm{~mL}$, or $6 \%$ of bladder capacity, can be defined as elevated $\mathrm{PVR}^{4)}$. However, a single estimation of PVR is not definitive for any case. Comparison of values during serial follow-up could be more reliable in determining whether a given PVR is abnormal. For example, even if a patient showed a single episode of elevated PVR, it may not be considered as abnormal if PVR is not elevated in previous measurements. Moreover, if there is an ample evidence for fecal impaction, the elevated PVR is more likely to be caused due to fecal impaction than neurological bladder dysfunction. Thus, if the patients showed only a single episode of abnormal PVR, it would be safe to there is no significant neurological change that affects voiding function. The limitation of PVR measurement is that it is the end-product of the entire voiding process, and several factors might affect this variable, such as water intake, structural abnormality of the urinary tract, anxiety, and fecal impaction. Repetitive measurement along with addressing the above-mentioned causes could be helpful in discerning the appropriate causal factor. Even if PVR is normal, it might be difficult to determine if the patient's voiding was normal. Some patients with meningomyelocele show normal PVR despite no discernible voiding. In this case, it is likely that the bladder neck was so incompetent that there is little chance of urine accumulation in the bladder.

The availability of a portable ultrasound scanner to measure the urine volume in the bladder has necessitated the catheterization to measure PVR, although its applicability in children has not yet been established.

\section{Uroflowmetry}

In the patients who have completed their toilet training, uroflowmetry is an adequate tool for evaluation of the emptying function. In this study, the patient voids into a funnel in which a sensor that produces a flow (volume-time) curve. It is usually combined with PVR measurement and is mainly used for nonneurogenic patients. When patients show bell-shaped good uroflowmetry results, with minimal PVR, there is no further need to check the voiding phase using an invasive urodynamic study (UDS).

\section{UDS}

UDSs are employed to evaluate the physiological parameters that are involved in bladder mechanics during filling and voiding. During the evaluation of neurogenic bladder, cystometry provides the most adequate information by measuring the detrusor pressure and sphincteric electrical activity. Cystometry simulates the filling and voiding phases by gradually filling the bladder. At the same time, the bladder and abdominal pressures are recorded by simultaneous measurement of both intravesical and rectal pressures, the latter of which is a proxy for intraabdominal pressure. By subtracting intra-abdominal pressure from intravesical pressure, one can accurately measure the genuine detrusor pressure during filling and voiding. The overall data from the fluoroscopic images or electromyography of pelvic floor muscles could provide insight into the sphincteric activity during the voiding as well as the filling cycle ${ }^{3)}$.

This helps us to better understand detrusor activity, bladder sensation, bladder compliance and capacity, and urethral function. Videourodynamic study that combines fluoroscopic imaging with conventional cystometry can provide better information on urethral movement, changes in bladder shape, and occurrence of vesicoureteral reflux ${ }^{3)}$. One drawback of this study is the invasiveness related to the need for catheterization. Therefore, UDS is not indicated for all patients, but should be considered when : 1) medical treatments do not work, 2) surgery that may affect urological function is scheduled, and 3) noninvasive study is not conclusive.

\section{GOAL OF UROLOGICAL EVALUATION}

Since most TCS-associated urological morbidities as well as mortalities are caused by renal dysfunction due to elevated bladder pressure, the primary objectives of urological evaluation involves early recognition of elevated bladder pressure or identifying the cases that would exhibit a high risk for changes. Adequate proactive treatment has been reported to be effective in preventing the development of renal injury and subsequent renal functional loss ${ }^{8)}$. Thus, identifying the patients who are likely to show deterioration is crucial. The secondary objective is to understand the state of the bladder and to provide proper management strategies for urinary incontinence and urinary tract infection. 


\section{PATHOPHYSIOLOGY OF UROLOGICAL DETERIO- RATION}

To achieve the above-mentioned goals, we should be familiar with the pathophysiology of neurogenic bladder caused by TCS. The neurogenic bladder can be categorized into four types according to the status of the bladder and the sphincter (Fig. 1) $)^{7,9)}$.

Since SD, such as meningomyelocele, typically affects the conus medullaris level, eliciting lower motor neuron disease ${ }^{5)}$, typical neurogenic bladder, inherent to SD, would exhibit low contractility of either the detrusor or external sphincter depending on the level of neural involvement. However, some cases of neurogenic bladder are characterized by detrusor overactivity (DO) and DSD, which indicates involvement at the suprasacral level. Although the precise reason for this has not yet been identified, the presence of TCS seems to be involved, putting a downward dragging tension on the neural segment, leading to disinhibition of supraspinal control. This might result in the release of primitive spinal reflexes, such as DO and DSD, both of which are suggestive of impaired supraspinal control. Infravesical obstruction, elicited by DSD, and maintenance of high vesical pressure, provoked by persistent DO, might induce ischemic changes in the bladder wall, including fibrosis and detrusor hypertrophy. In addition, persistently elevated PVR and concurrently occurring bowel dysfunction make the bladder vulnerable to urinary tract infection. During decompensation, trabeculation of the blad- der wall becomes aggravated and deformation of the ureterovesical junction leads to either incompetence (vesicoureteral reflux) or obstruction. The resultant backflow of urine to the kidney causes hydronephrosis and renal parenchymal damage due to blockage of urine. When urine is contaminated by bacteria, the risk of renal parenchymal invasion by bacteria is elevated, accelerating further renal damage.

\section{EVALUATION BEFORE THE UNTETHERING SUR- GERY}

Fig. 2 shows the flowchart of the perioperative evaluation process. Although preoperative UDS may not be supported in some cases and sometimes not feasible in others with open SD because of difficulty in placing patients in the supine position, preoperative bladder function might be helpful for postoperative care. In patients showing synergic voiding during preoperative UDS, it is unusual if patients cannot void properly. For neurosurgeons, it is discerning when a child, who is seemingly able to void normally, cannot void spontaneously postoperatively. If normal preoperative voiding function is confirmed, the postoperative voiding difficulty might be related to intraoperative or postoperative neural damage.

For these patients, basic evaluation should include renal ultrasound and PVR assessment. Renal ultrasound should be checked at baseline because $5-10 \%$ of newborns have been reported to exhibit an abnormal urinary tract on radiologic ex-

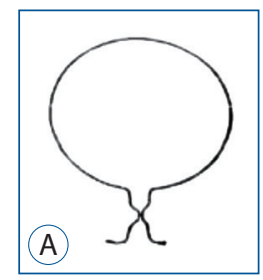

Dysfunction

A : sphincter ++ / detrusor --

B : sphincter ++ / detrusor ++

C : sphincter -- / detrusor --

D : sphincter -- / detrusor ++

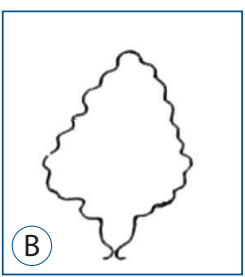

Consequences unsafe, leaking infections DSD, unsafe from birth (reflux, infections, renal damage) safe but wet

wet and unsafe
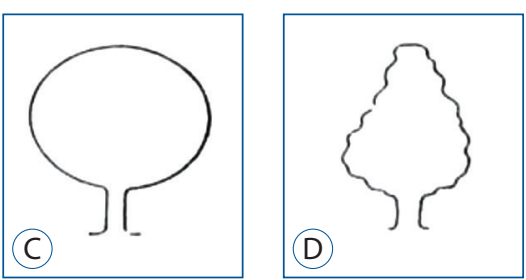

Management

safe and dry with CIC

safe and dry with anticholinergics+CIC

safe and dry with CIC+outlet surgery can occur detrusor overactivity after outlet surgery

safe and dry with $\mathrm{CIC}+$ anticholinergics +outlet surgery

Fig. 1. Urodynamic assessment classifies a neurogenic bladder into four types. Relevant consequences and management are also described. In accordance with the sites of dysfunction, four subtypes can be identified. A : Both sphincter and detrusor are underactive. B : The sphincter is overactive and detrusor is also overactive. C : Both sphincter and detrusor are underactive. D : Sphincter is underactive but detrusor is overactive. Adopted from Verpoorten and Buyse ${ }^{9)}$. DSD : detrusor sphincter dyssynergia, $\mathrm{CIC}:$ clean intermittent catheterization. 


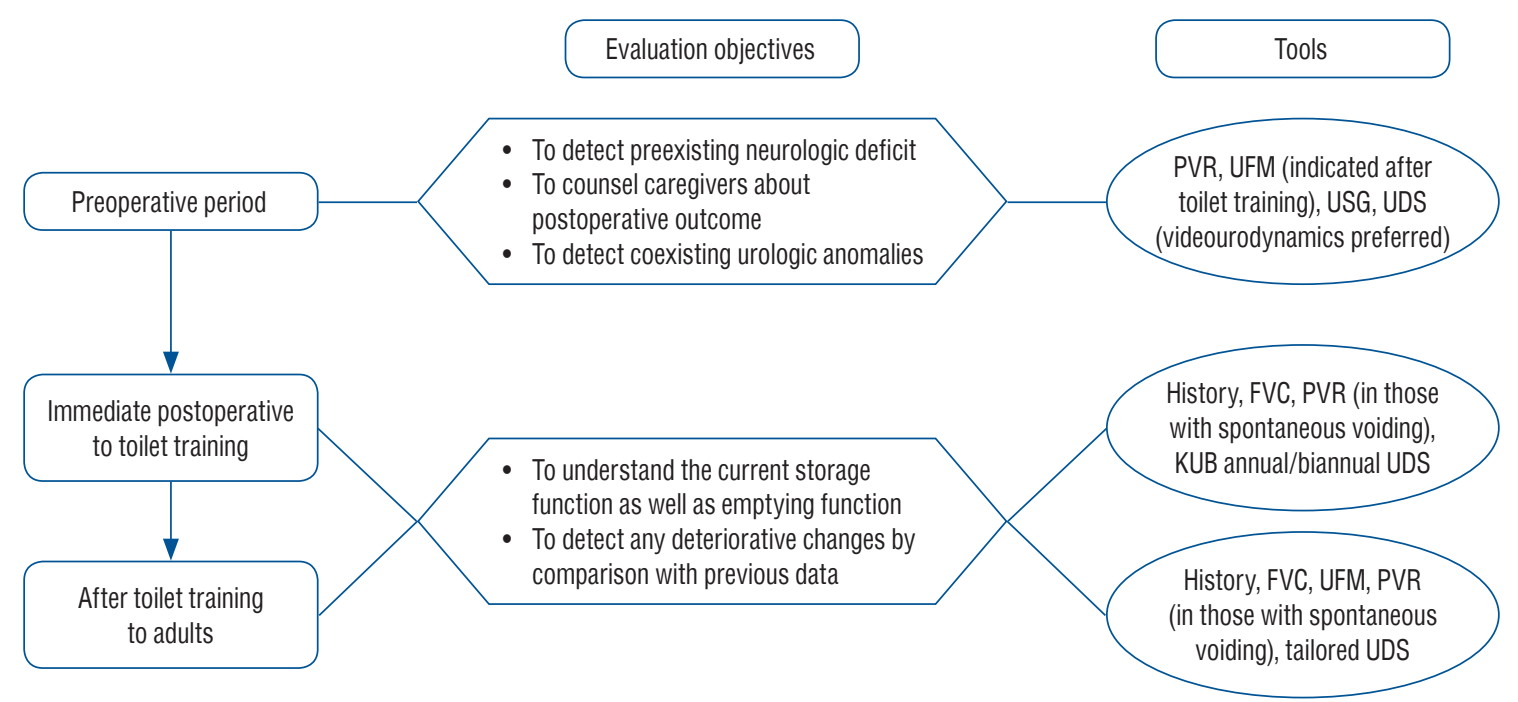

Fig. 2. Follow-up protocol applied to patients after initial untethering to adults. Due to various manifestations and time courses, there is no "one size fits all" follow-up scheme and evaluation process should be tailored according to the conditions. For rationale and detailed scheme, please refer to the manuscript. PVR : postvoid residual urine, UFM : uroflowmetry, USG : ultrasonography of kidney and bladder, UDS : urodynamic study, FVC : frequency volume chart, KUB : simple radiography of kidney, ureter and bladder.

amination. The presence of hydronephrosis, hydroureter, and thickened bladder wall indicates the presence of potential urinary tract anomalies related to renal damage unless they are managed properly. In infants, PVR can be measured after the voiding in diapers. Parents must be asked to check their child's diaper every 30 minutes, to avoid missing the timing of excessive voiding. Normal voiding is usually associated with minimal PVR (less than 10\% of the estimated bladder capacity). Thus, minimal PVR suggests good emptying ability, excluding the possibility of DSD and poor detrusor contractility. However, there are some caveats. Normal PVR may occasionally be seen in cases with sphincteric incompetence.

The status of the bladder can be confirmed using UDS, especially cystometry. Patients with lower motor neuron dysfunction, caused by SD, typically show weak detrusor contractility and/or sphincteric incompetence. However, they might also exhibit DO, DSD, or signs of upper motor neuron disease.

This is either due to the presence of TCS or is the sign of immaturity, suggesting incomplete control of the spinal reflex. In infants, especially premature children, it is not uncommon that upper motor neuron signs are seen even in neurologically normal children. Thus it is important to discern whether these findings are truly pathological. There are no definite criteria to differentiate this enigma; however urologists should gather all potential clues for confirmation, such as age, history of PVR, concomitant UDS findings, and potential presence of TCS, based on opinions from neurosurgeons. In a normal child, discordant voiding, contrary to neurological DSD, seems to undergo transition to normal synergic urethral movement within 6 to 9 months of age. Therefore, prominent DSD in patients older than 1 year indicates the presence of TCS. On the other hand, presence of only DO, without any other evidence for DSD, is unlikely to be associated with TCS in young children (less than 6 months old), especially when their PVR is normal.

\section{EVALUATION AT THE IMMEDIATE POST-UNTE- THERING PERIOD}

During the immediate postoperative period, most children may not void well due to temporary neurapraxia. This usually lasts for several days or even months ${ }^{2}$. However, most patients recovered their emptying ability within 1-2 months, normalizing their PVR. To prevent urinary retention, which is detrimental to the recovery of bladder contractility, prolonged indwelling of the urethral catheter or CIC could be performed. Among these, the latter is thought to be better during postoperative recovery, for controlling urinary tract infection and preventing urethral injury. Thus, parents must be educated to do CIC. In cases of persistent elevated residual urine for more 
than 2 months, prolonged CIC might be required, but some parents may not follow the recommendation and let the child try spontaneous voiding in the hope that voiding training would restore the emptying ability. This is not recommended because voiding, which requires coordination of the autonomic nervous system, may not be trained. For example, the adult patients who have lost their voiding function after radical pelvic surgery or spinal surgery do not have any sensation in the bladder that could be trained. In the case of children, perception of this sensation is more difficult. In order to help in the recovery of emptying function, administration of an alpha antagonist or control of fecal impaction might be considered, although their benefits have not yet been established.

Even after several months of surgery, patients may not void appropriately. The possible reason may be significant operative damage to neural tissue or development of retethering. The former may gradually redevelop their sensation and reestablish spontaneous voiding a few years later, whereas the latter might result in progressive deterioration of the bladder along with an inability to void.

Since all patients, except those that undergo retethering, exhibit gradual improvement over time, the main purpose of urological follow-up is to check for bladder deterioration. The patients who can void spontaneously must monitor their PVR and perform simple abdominal radiography. These tests examine the efficiency of voiding and defecation, which might explain the reason for elevated PVR. For those who undergo intermittent catheterization, their parents may be requested to fill in the frequency volume chart as a measure of the storage function of the bladder. Upper urinary tract evaluation using ultrasound may be performed every year until the patients achieve toilet training with stable bladder function. The interval of UDS evaluation can be tailored according to the risk of bladder deterioration. In those who are able to void spontaneously, follow-up UDS may safely be skipped in the case of normal PVR and upper urinary tract. In those who required CIC, follow-up UDS should be performed every year, but the interval may safely be extended in case of increase in storage function of the bladder and normal upper urinary tract. Prompt evaluation using UDS should be considered in cases exhibiting progressive decrease in bladder capacity, unexplained urinary incontinence, or recurrent urinary tract infection.

\section{TOILET TRAINING}

From a developmental perspective, toilet training should be regarded as a cornerstone event during urological follow-up. This means that the child must know how to urinate and defecate. In other words, the baby must be able to sense the filling and emptying of the bladder and bowel. Usually, adequate bowel control should precede bladder control. Although some patients with inherent bowel issues (like imperforate anus that is often associated with TCS) might show reversal of this sequence, that is, fecal incontinence persists despite adequate urinary control, it is natural that urinary continence should follow fecal continence. Thus, when patients show a reversed sequence of urinary and bowel control during toilet training, the presence of constipation should be assessed. Patients with SD often show delays in accomplishing toilet training. This is often perceived as the case due to the incomplete development of neural integrity and maturation attributed to these groups of patients. However, unlike the patients who failed to void spontaneously and required CIC due to overt neurological dysfunction, those who showed spontaneous voiding exhibit comparatively mild and reversible neurological problems. The most effective way is to relieve fecal impaction which is common in these patients and prohibits them from achieving toilet training. Active treatment of fecal impaction is expected to facilitate normal toilet training without significant delay.

Among the patients who can void spontaneously, progressively increasing amounts of PVR and failure to show any improvement with time might indicate secondary reoccurrence of TCS. In such case, first, active fecal control should be performed initially. Those who fail to show any improvement should be evaluated using UDS and treated accordingly.

\section{DETECTION OF DETERIORATION OF BLADDER STATUS DURING CHILDHOOD AND ADOLES- CENCE}

Deterioration of the bladder is not uncommon in patients with TCS. Although the mechanism of this deterioration has not been elucidated, chronic overdistension of the bladder, associated with infravesical obstruction (due to DSD) and persistent DO, is thought to play a key role in detrusor hypertrophy and fibrotic changes. Furthermore, failure in removing 
the intruding bacteria could contribute to detrusor changes due to chronic inflammation. Early detection and proper management would alleviate this process. Prevention of chronic bladder distention by intermittent catheterization and management of DO with anticholinergics has been advocated to prevent deterioration. Although UDS is the best option to measure the changes in bladder accurately, it cannot be applied to all the patients because of its invasiveness and cumbersomeness. In this regard, measurement of PVR and/or uroflowmetry could be used as a good alternative for UDS. Given that PVR can be considered as the end result for emptying function and DSD is the cardinal urological manifestation of TCS, PVR assessment can be used for detecting urological abnormality in TCS patients. However, one caveat is that PVR can be affected by several factors, such as subjective mood, unusual circumstances, and most importantly, fecal impaction, even in toilet-trained children. Thus, careful scrutiny is required while interpreting PVR and performing UDS.

Since TCS-associated urological deterioration can occur at any time during follow-up, urologists should be responsible for examining these patients at regular intervals. Surveillance for these patients should be tailored according to the risk of deterioration. The occurrence of TCS is assumed to be related to linear growth, which could put linear dragging tensions on either side, and hence, the patients should be at least followedup until the completion of puberty. However, a few patients showed overt deterioration even after the cessation of linear growth. In addition, according to the experiences of adult patients with primary TCS, it is unusual for patients to realize the occurrence of their first symptom in adulthood. Thus, it is prudent to monitor these patients life-long. In those who are capable of spontaneous voiding, patients are annually or biannually followed-up until puberty, and are informed of abnormal voiding habits that are related to deterioration of the bladder. Spontaneous voiders have better sensation than those requiring CIC, so their urinary problems can easily be seen when they have been informed of possible symptoms.

Before the toilet training, however, their status should be monitored via PVR assessment. In case of elevation of PVR, control of fecal impaction, which is common in these patients, might be required.

On the other hand, patients with CIC are more likely to exhibit bladder deterioration. The deterioration of the bladder can be attributed to a history of frequent urinary tract infec- tions and recently aggravated urinary incontinence. In addition, aggravation of other systems, such as uncontrolled fecal incontinence or constipation and progression of orthopedic abnormalities should be included as a potential factor that indicates bladder deterioration. Patients should be requested to keep a frequency volume chart to assess the volume that they drain during CIC, which best approximates the storage function of their bladder. If they persistently show smaller bladder volume than the age-estimated value, it indicates that they have some bladder problems.

Before examining patients using UDS, it should be made sure whether CIC is well-followed and anticholinergics are properly administered. Since they have less sensation of bladder filling, negligence of CIC or noncompliance of drugs might transiently lead to deterioration of the bladder due to chronic overdistension of the bladder and uninhibited DO. However, when they show significant deterioration of the bladder despite adequate management (CIC and anticholinergics), it might indicate presence of TCS.

Since DSD is the cardinal manifestation of TCS and should be revealed during UDS, the use of fluoroscopic imaging or needle electromyographic study is definitely helpful. Although there are no reported cardinal features of DSD, active contraction of the external sphincteric area or hyperactive EMG in response to increasing bladder pressure can be considered as DSD.

\section{CONCLUSION}

Patients with SD exhibit a wide range of risks for progression to TCS. Even after successful untethering, there is a continuing risk of urinary tract damage. Given that urological morbidity is common and critical to life, urologists should be responsible for managing the patients and cooperating with neurosurgeons. Measuring and interpreting PVR values in the context of development is important. UDS is important in such patients because some patients with upper motor neuron signs show deterioration of the bladder and upper urinary tract due to TCS. Urologists should reveal the risk of the urinary tract deterioration, provide a strategy to manage the problems, and discuss helpful information with neurosurgeons to allow the patients to benefit from untethering at appropriate time. 


\section{CONFLICTS OF INTEREST}

No potential conflict of interest relevant to this article was reported.

\section{INFORMED CONSENT}

This type of study does not require informed consent.

\section{AUTHOR CONTRIBUTIONS}

\author{
Conceptualization : KP \\ Data curation : KP \\ Writing - original draft : KP \\ Writing - review \& editing : KP
}

\section{ORCID}

Kwanjin Park https://orcid.org/0000-0002-8926-3047

\section{References}

1. Austin PF, Bauer SB, Bower W, Chase J, Franco I, Hoebeke P, et al. : The standardization of terminology of lower urinary tract function in children and adolescents: Update report from the standardization committee of the International Children's Continence Society. Neurourol Urodyn 35 : 471-481, 2016

2. Bauer SB, Austin PF, Rawashdeh YF, de Jong TP, Franco I, Siggard C, et al. : International Children's Continence Society's recommendations for initial diagnostic evaluation and follow-up in congenital neuropathic bladder and bowel dysfunction in children. Neurourol Urodyn 31 : 610-614, 2012

3. Bauer SB, Nijman RJ, Drzewiecki BA, Sillen U, Hoebeke P; International Children's Continence Society Standardization Subcommittee : International Children's Continence Society standardization report on urodynamic studies of the lower urinary tract in children. Neurourol Urodyn $34: 640-647,2015$

4. Chang SJ, Chiang IN, Hsieh CH, Lin CD, Yang SS : Age- and gender-specific nomograms for single and dual post-void residual urine in healthy children. Neurourol Urodyn 32 : 1014-1018, 2013

5. Light JK, Faganel J, Roth DR, Dimitrijevic MR : Meningomyelocele: a clinical, urodynamic and neurophysiological evaluation. J Urol 131 : 717-721, 1984

6. MacLellan DL : Neuromuscular dysfunction of the lower urianry tract in children in Wein AJ, Kavoussi LR, Partin AW, Peters C (eds) : CampbellWalsh urology. Philadelphia : Elsevier, 2016, Vol 4

7. Madersbacher $\mathrm{H}$ : The various types of neurogenic bladder dysfunction: an update of current therapeutic concepts. Paraplegia 28 : 217-229, 1990

8. Stein R, Bogaert G, Dogan HS, Hoen L, Kocvara R, Nijman RJM, et al. : EAU/ ESPU guidelines on the management of neurogenic bladder in children and adolescent part I diagnostics and conservative treatment. Neurourol Urodyn $39: 45-57,2020$

9. Verpoorten C, Buyse GM : The neurogenic bladder: medical treatment. Pediatr Nephrol 23 : 717-725, 2008 